\title{
Perspective and general approach of diabetes in palliative care
}

\begin{abstract}
The progressive aging of the population and the increase in chronic diseases means that we are facing more and more patients who require palliative care. It is necessary to reinforce the multidisciplinary work and the coordination between first level care and hospital care. In addition to changes in health policies, it is required to train health professionals more in communication skills and in the ethical and humanitarian perspective of the care of patients in the process of dying. For patients with diabetes, adequate assessment of their prognosis and the adequacy and management of their medications should be reinforced in all patients who enter the care phase at the end of life to minimize the side effects and complications derived from them. Medications and maintain comfort and quality of life as best as possible. Properly communicate changes to family members and patients to decide together the best treatment strategy.
\end{abstract}

Keywords: diabetes, poly-pathology, palliative, terminal care, advanced treatment plan
Volume 2 Issue 3 - 2018

\author{
Díaz Rodríguez Juan Javier ${ }^{1,2}$ \\ 'Department of primary health care of chronic disease, Merida \\ Health Center, Health Services of Yucatán, México \\ ${ }^{2}$ Director of Center of education and research on chronic \\ disease (Educady), México
}

\author{
Correspondence: Díaz Rodríguez Juan Javier, Department \\ of primary health care of chronic disease, Merida Health \\ Center, Health Services of Yucatán, Calle $50 \# 330$ x 53b, \\ Fraccionamiento Fco de Montejo, Mérida Yucatán. México, CP \\ 97203, Tel +52-I-999-9-46-9|-|8, \\ Email javier.diaz@cady.com.mx, \\ centrodiabetesmerida@gmail.com
}

Received: May 29, 2017 | Published: June 22, 2018

\section{Objective}

The objective of this review is to provide an overview of palliative care in patients with diabetes. Describe the relationship between diabetes and co-morbidity, its impact on the prognosis and the different strategies to carry out the treatment plan for patients who enter the end-of-life scenario.

\section{Introduction}

Diabetes is a major health emergency of the 21 st century; It is estimated that 425 million people worldwide have diabetes and it is expected that by 2045 they will be approximately 693 million. ${ }^{1}$ It is a chronic disease that is associated with multiple complications derived from chronic hyperglycemia and is related to various comorbidities. ${ }^{2,3}$ Also, diabetes is connected to an increased risk for the development of some types of cancer. ${ }^{2,4}$

Diabetes is among the ten leading causes of death worldwide ${ }^{1}$ considering that there is a death from diabetes every 6 seconds in the world 5 and conjunction with the cardiovascular diseases, cancer, and respiratory diseases, represents more than $80 \%$ of deaths from noncommunicable diseases. ${ }^{1}$ There is a vast literature that relates to diabetes with an increase in morbidity and premature death ${ }^{6}$ reducing life expectancy up to 6 years in people over 50 compared to people without diabetes ${ }^{7}$ and, although a minority of people die early, up to $90 \%$ of people die after a long evolution of the disease ${ }^{8}$ Being, in the higher percentage, deaths related to cardiovascular and renal complications; and only a small rate of up to $3 \%$ is directly related to the disease. ${ }^{6}$ Although cardiovascular disease is not a single entity in diabetes, it accounts for up to $75 \%$ of deaths in patients with diabetes, ${ }^{6}$ it has been corroborated by multiple studies that mention that diabetes doubles or quadruples the risk of cardiovascular disease. ${ }^{9,10}$ In a recent study, it was found that individuals with diabetes compared with those without diabetes were at high risk of death from cardiovascular disease, dying from cancer, and death from any cause. ${ }^{11}$
The world population is aging, ${ }^{5}$ either due to a higher availability of effective treatments or the progressive aging of the population, at present people with chronic conditions that condition disability are increasing, and this represents a significant challenge to be able to maintain an adequate quality of life in these people. ${ }^{12}$ The incidence of diabetes increases with age, and as a result, a large proportion of people over 65 will have diabetes; either of recent diagnosis or longstanding diabetes. ${ }^{13}$ According to data from the International Diabetes Federation, more than $35 \%$ of older adults have diabetes, and it is considered that 20 to $30 \%$ have prediabetes..$^{5}$ This high prevalence is related to physiological changes that lead to an increase in insulin resistance and impaired pancreatic function. Older adults with diabetes are more at risk of developing complications, related to amputations, end-stage renal disease, and myocardial infarction compared to other age groups. This fact makes them a very heterogeneous group regarding disease and functional impairments. ${ }^{13,14}$ All this makes diabetes a considerable burden for self-care over time and that the needs of endof-life care are very complicated for people with diabetes. ${ }^{3}$ To a load of self-care skills related to diabetes, ${ }^{15}$ subsequent deterioration is added to other associated comorbidities and complications of diabetes itself. ${ }^{3}$ Also, in older adults, the presence of geriatric syndromes that include frailty falls, depression, incontinence and polypharmacy increases. $^{14}$

The implementation of a palliative care plan at an early stage, even integrated into the usual diabetes care plan, can prepare the patient and his family members for the necessary changes in treatment when the end of life approaches. ${ }^{5,6}$ Thus, Palliative care can have a positive effect on the disease, function, quality of life and the care burdens derived from care and treatment. ${ }^{5}$ However, the notion of palliative care and its integration into the self-care program of patients with diabetes are not considered by many health providers. ${ }^{6}$

\section{Diabetes and its diverse trajectories}

Palliative care originally refers to end-of-life care. ${ }^{5}$ During which a care designed to provide well-being or comfort is granted, as well as 
support to patients and their families in the final stages of a terminal oncological or non-oncological disease. Originated in the Middle Ages in the so-called Hospices comes from the word "pallium" which means mantle or cover, alluding to the act of curing symptoms or suffering. ${ }^{16}$ It is necessary for healthcare providers to know the definitions of palliative care, end-of-life care and terminal care (Table 1). ${ }^{5}$ Since diabetes is a common condition in the framework of palliative care, ${ }^{12}$ and that can present an unpredictable trajectory, which can lead to missed opportunities to discuss palliative care and, also, lead to interventions unnecessary at the end of life. ${ }^{5}$

Table I Definitions

\begin{tabular}{ll}
\hline & $\begin{array}{l}\text { It refers to granting a care designed to provide well- } \\
\text { being or comfort and alleviate suffering in people } \\
\text { treated with incurable diseases to improve their quality } \\
\text { of life; as well as support to patients and their families } \\
\text { in the final stages of a terminal oncological or non- } \\
\text { oncological disease }\end{array}$ \\
$\begin{array}{l}\text { Palliative care } \\
\text { of life }\end{array}$ & $\begin{array}{l}\text { It refers to the care of people who will die in the next } \\
\text { Inonths }\end{array}$ \\
\hline
\end{tabular}

Palliative care for people with diabetes can be more demanding for many reasons: they generally require more extended hospitalization compared to people without diabetes, they present greater clinical instability that makes it necessary to need more time for clinical assistance compared to non-diabetics in their glucose monitoring as in the time used to carry out patient and caregiver education; there is a higher prevalence of complications, and comorbidities, as well as the associated polypharmacy; all this increases the risk of complications related to treatment and interactions related to hyperglycemia and hypoglycemia. ${ }^{3,12}$ Consider that diabetes ${ }^{14}$ and many chronic diseases associated with death occur at older ages. ${ }^{3}$ However, the prevalence of type 2 diabetes in young people has increased, ${ }^{3,17}$ this leads to the development of complications and death at younger ages. ${ }^{3}$ The trajectories of the disease correspond, in general, to different rates.

The evolution and priorities of care that are very useful for categorizing the state of health and establishing the most appropriate care. ${ }^{18}$ People with diabetes can follow any of these trajectories. ${ }^{3}$

Three trajectories have been described by most of the experts. ${ }^{3}$ A trajectory of rapid deterioration, often known as the trajectory of cancer and characterized by a clinical deterioration that can go weeks, months or years. ${ }^{18}$ Diabetes is related to the incidence of multiple types of cancer ${ }^{19}$ where hyperglycemia and hyperinsulinemia [20] are factors, the latter being the most consistent factor related to the development of neoplasms. ${ }^{21}$ Sudden death has a significant impact especially when death is unexpected. ${ }^{5}$ Chronic disease trajectory, in which patients tend to live for a relatively long time but which is characterized by relapses or exacerbations due to the loss of their functional reserve and other associated comorbidities, with death occurring for some of them. ${ }^{18}$ The unpredictable evolution of diabetes leads to episodes of de-compensation due to intercurrent or complication-related diseases, ${ }^{3}$ mainly hyperglycemic crises $^{22}$ or hypoglycemia. ${ }^{23}$ Although individuals survive most of these episodes, there is clinical deterioration after each of them, ${ }^{3}$ and death may be imminent in some of them. ${ }^{16}$

The trajectory of prolonged fragility, those people who survive cancer or other conditions develop deterioration of functional reserve over time and die at older ages. In this group, Alzheimer's disease or other dementias are characterized. ${ }^{3,5,18}$ Fragility is characterized by a decrease in the physiological reserve that conditions vulnerability to adverse events, often associated with the aging process ${ }^{24}$ and is a critical factor in the prognosis of many chronic conditions. ${ }^{25}$ Older adults with frailty and diabetes are more vulnerable to the adverse effects of medications such as hypoglycemia and other conditions that must be considered such as falls syndrome, polypharmacy, impaired renal function, cognitive alterations, visual deficits, and social conditions such as living alone, ${ }^{26}$ malnutrition and sarcopenia are also present, ${ }^{27}$ which reduces their quality of life. ${ }^{25}$ Therefore makes the treatment of diabetes in older adults represents important challenges that require an assessment of barriers and challenges to achieve adequate treatment reducing the risk of adverse events. ${ }^{14}$ Dunning et al., suggest a new category, which they call Health, in its people are healthy, self-dependent and do not present complications. The priority is the strict control of glycemia and healthy lifestyles to prevent complications. ${ }^{3}$

\section{Identifying patients with diabetes that require palliative care}

Palliative care is defined as an approach that improves the quality of life of patients and families living with a life-threatening disease. The objective is prevention, alleviating suffering through the early identification of problems through an evaluation and encouraging the participation of the individual and caregivers in decision-making. ${ }^{28}$ Managing diabetes is a challenge, especially when it comes to end of life. ${ }^{5,29}$ Considered as a difficult period in which the management varies significantly and with different phases of progression towards death; which can range from rapid progress to end to long periods of relapse. ${ }^{30}$ The above-mentioned leads to difficulties in the decision making of health providers and problems to implement appropriate strategies and management plans. ${ }^{29}$ There is evidence that doctors are not proactive in addressing the issues of palliative care, but until very advanced stages of the disease. ${ }^{31}$

Such difficulty afore-said is observed regardless of social or ethnic status and has been corroborated for diabetes, chronic kidney disease and any of the chronic conditions not related to cancer, and is often derived from the use of different definitions of care at the end of the life, which allows to adequately discuss how and when to initiate or not the necessary interventions. ${ }^{32}$ The best time to start palliative care is when people are in a stable disease phase with the ability to make informed decisions. ${ }^{5}$ It should be done early, even from the diagnosis of diabetes and not only in those close to death..$^{33}$ However, palliative care and end-of-life care are not considered by most of the guidelines of diabetes guidelines as part of the usual care of patients. ${ }^{8}$ The main guidelines only refer to the need to start this type of care in older adults. $^{34}$

End-of-life care includes people who are likely to die within the next 12 months and include imminent death (death within a few hours or days) and incurable diseases, frailty, acute illnesses as well as preexisting conditions that can cause sudden death like diabetes. The Gold Standards Framework (GSF) describes a color code that divides five scenarios that allow to more accurately assess the specific needs of people to carry out care strategies and coordinate more effectively the care of patients (Table 2). ${ }^{35}$

It must be considered that, these stages are dynamic, and that they can overlap during diabetes as complications of the same or acute diseases that worsen the prognosis of patients, and impair their 
functional reserve, and although the GSF does not specifically mention diabetes, it is a useful tool when planning care strategies at the end-oflife care ${ }^{5,29}$ The Gold Standards Framework Proactive Identification Guidance (GSF PIG) establishes a guide to help health providers identify people who need end-of-life care and develop an action plan considering the prognosis; anticipating the probable deterioration and discussing the needs of patients early in collaboration with patients, relatives or caregivers. It describes three key steps that include: proactive identification of patients who may be in the last year of life, assessing their current and future needs, and establishing a plan.

Table 2 Prognostic scenarios of The Gold Standards Framework

$\begin{array}{ll}\text { A - Blue } & \text { Stable patients (Prognosis of years) } \\ \text { B - Green } & \text { Unstable and advanced disease (Month forecast) } \\ \text { C - Yellow } & \text { Continuous care (Weekly forecast) } \\ \text { D - Red } & \text { Final days of life (Death in days or hours) }\end{array}$

The strategy suggests as an initial step a trigger question called "surprise question" that serves to identify those patients who are about to die: "Would you be surprised if the patient die in the next year, month or days?" If the answer is NO, the process of identification, evaluation and action plan begins. This requires a thorough evaluation that includes two more steps. The first identifies the possible increase or decrease of specific needs and the next one is focused on the evaluation of three trajectories that include characteristics related to cancer, failure of different organs (such as heart disease, chronic obstructive pulmonary disease) as well as frailty. ${ }^{36}$ This framework promotes an assessment and treatment plan focused on the person that allows health providers, patients and family members and / caregivers to work collaboratively in a treatment plan adapted to the needs of the person.

\section{Diabetes and multimorbidity}

The presence of diabetes is usually associated with multimorbidity; ${ }^{12}$ this, increases with age being more prevalent in people older than 65 years ${ }^{37}$ However, it can have a high impact and costs in younger patients ${ }^{38}$ as demonstrated in one study, where more than half of patients younger than 65 years had multimorbidity. ${ }^{37}$ There is evidence that shows that this condition has a remarkable impact on the prognosis. These patients die more prematurely, estimating an annual mortality in primary care of $6 \%$, and of $19-20 \%$ in the hospital setting, and up to $36 \%$ a year, ${ }^{39}$ they have more income, and more extended stays, in addition, their quality of life is reduced, as well as their functionality, and they present more episodes of depression compared to patients without multimorbidity. ${ }^{38}$

The context of patients who are integrated into palliative care, and at the end of life care is that of multimorbidity ${ }^{3}$ and is a common clinical phenomenon in diabetes. ${ }^{40}$ In this context, clinicians present difficulties both for the management of this patients ${ }^{41}$ and for establishing the necessary changes in end-of-life care, especially for making decisions to suspend or maintain interventions; creating difficulties often of an ethical nature and being especially difficult when the individual desires of the people were not documented. These dilemmas also include those related to treatments that negatively impact on the control of diabetes and spiritual needs. ${ }^{29}$ The GSF PIG, considering the assessment of multimorbidity in its evaluation process. ${ }^{36}$
On the other hand, given the need for a more specific assessment of the needs of patients with multiple pathologies, a group of researchers developed a tool that assesses comprehensively. This tool, called PROFUND, predicts in-hospital mortality at 12 months $^{41}$ and has been valid for use in primary care. ${ }^{42}$ It aims to determine in a closer way the prognosis of patients with multimorbidity to structure a personalized action plan; avoiding over-treatment and unnecessary waste of resources. In such a way that, although it is not a specific tool for the end-of-life care plan, it is possible to discuss palliative care actions early in those people with poor prognosis; also identifying their needs and given their decisions as well as their spiritual and emotional aspects within a collaborative framework. ${ }^{41}$

\section{Establishing the personalized action plan}

It is necessary to provide multidisciplinary care by improving effective communication to avoid fragmented attention to the care of patients at the end of life. ${ }^{43}$ Care includes a strategy where communication between different members of the health team works collaboratively with patients and family members to reach informed agreements and at the same time recognizes emotional comprehension through generating empathy in the face of worries, pain, the anguish or the suffering of others. ${ }^{44}$ Many professionals find it difficult to communicate with other professionals and with patients. ${ }^{43}$ Therefore, it is necessary to equip them with the required skills for this which can be implemented even from the academic institutions. These should include not only clinical skills but also emotional and communication skills. ${ }^{44}$

End-of-life care includes the patient, family, friends and multiple health professionals where communication is the central capacity; ${ }^{3}$ decision-making must be recognized by the abilities of all involved members. ${ }^{44}$ The communication should ensure that family members can handle the situation and discuss the options proactively seeking the empowerment of the patient and the family. ${ }^{2}$ The attention must be personalized according to the needs and desires of the individual. ${ }^{29}$ This is especially relevant, since patients and families may perceive that the modification in the work is due to a little interest or even abandonment by health professionals. Ideally, these actions should be carried out as soon as possible so that the company can participate in the advance action plan and even in writing the advance documents when it is not possible. Although in many cases it is difficult for the family, even when making decisions with patients with health problems. $^{5}$

Once the person approaches the end of his life, there are several elements to consider. ${ }^{45}$ Blood tests such as blood glucose monitoring may be irrelevant or may require reducing its intensity. ${ }^{46}$ The control goals are adjusted according to the needs of the person. ${ }^{47,48}$ Insulin regimens should be modified to avoid rigorous schedules and medications adjusted to prevent hypoglycemia and osmotic symptoms. ${ }^{29}$ The management of drugs that could affect the control of diabetes should be considered especially corticosteroids since the use of these is frequent in several terminal diseases. ${ }^{2}$ Both patients and caregivers should receive the necessary counseling to understand the changes in treatment. ${ }^{29}$

\section{Changes and goals in the treatment plan}

The approach and changes of treatment and therapeutic management in end.-of-life care patients should be aimed at assessing patient needs, providing information and guiding patients and families 
to be able to improve the outcomes of diabetic patients as much as possible. ${ }^{49}$ Diabetes often coexists with other terminal diseases. The treatment must be adjusted to each patient. ${ }^{45}$

\section{Glycemic control}

The main objective of diabetes treatments is glycemic control. ${ }^{47}$ There is no consensus about glycemic goals in end-of-life patients ${ }^{29}$ but it is considered that blood glucose levels measured in blood should be maintained between $108 \mathrm{mg} / \mathrm{dl}(6 \mathrm{mmol} / \mathrm{L})$ and less than $275 \mathrm{mg} /$ $\mathrm{dl}(15 \mathrm{mmol} / \mathrm{L})^{48}$ and, although it has been considered irrelevant by many authors, ${ }^{50}$ there are studies that identify that an $\mathrm{HbAlc}$ value above or below $7.5 \%$ is a prognostic indicator for patients with cancer and type 2 diabetes who enter hospices; ${ }^{50}$ so a goal of HbA1c of more or equal $8 \%$ can be taken into account. ${ }^{47}$ These values would allow avoiding hypoglycemia or osmotic symptoms due to hyperglycemia in this type of patients. ${ }^{45}$

Hypoglycemia is the main limitation in the treatment ${ }^{47}$ and, although it can be defined in different ways, ${ }^{23}$ we consider that a patient with diabetes presents hypoglycemia when the glucose level it is below $70 \mathrm{mg} / \mathrm{dl}(3.9 \mathrm{mmol} / 1)$. The presentation of symptoms, in diabetes, is closely related to the degree of deterioration of the counterregulatory mechanism in patients with diabetes. ${ }^{51}$ It should be considered that older patients have an increased risk of hypoglycemia ${ }^{23}$ related to multiple factors such as deterioration of renal function, increased deterioration of the counterregulatory mechanism, polypharmacy, frailty and appetite alteration. ${ }^{14}$ In the scenario of patients in end-oflife care, the risk is substantial due to its association with anorexia, nausea or vomiting and reduced quality of life. ${ }^{29}$ It is related to increased mortality and is the most significant metabolic threat in patients close to death. ${ }^{50}$ It is associated with increased use of hospital services and cognitive impairment. ${ }^{51}$ Also, patients with end-of-life care may present with liver and kidney failure, which impacts on the dose and management of drugs ${ }^{49}$ considering that the combination of different hypoglycemic drugs and the use of Insulin are risk factors for hypoglycemia. ${ }^{23}$

On the other hand, monitoring the development of hyperglycemia is necessary to avoid the complications of polyuria, polydipsia, nausea and dehydration that accompany it; which reduces the quality of life of patients. ${ }^{43}$ Monitoring is essential in these cases as well as training the caregivers to identify the symptoms and carry out the treatment strategies. ${ }^{29}$ The monitoring of blood glucose is essential to achieve the goals of control ${ }^{47}$ although in the end-of-life care must adjust to the needs of patients or even suspend it in patients in the process of dying driving the urinalysis optionally and leaving the blood glucose control for those symptomatic patients. ${ }^{2}$ It is important to comment on these changes to patients, relatives, and caregivers ${ }^{46}$ since they are often accustomed to strict monitoring, and these actions can be taken as an attitude of little interest by health professionals. ${ }^{29}$

The management and adaptation of medications or metabolic control in patients at the end of life, especially in patients ready to die is included by multiple characteristics, which hinders the actions of medical professionals who in many cases are not trained for the approach of that type of patients..$^{48}$ The steps required to prescribe and depress must depend on the availability and cost of the medications, the patient's prognosis, the state of health, their capacity to administer them orally and associated comorbidities. ${ }^{46}$ The coding in four scenarios of The GSF can be useful to decide the necessary changes in the adaptation of medicines (Table 2). ${ }^{48}$

\section{Patients with type I diabetes}

The etiology of type 1 diabetes is the autoimmune destruction of beta cells, and the specific treatment is with the application of insulin dose. ${ }^{52}$ There are no randomized controlled studies about the management of patients with type- 1 diabetes at the end of life..$^{50}$ Patients with type 1 diabetes can continue with insulin treatment even in the final stages as these patients are at high risk of ketoacidosis even when they stop eating. ${ }^{50}$ It may be necessary to use simplified schemes, reduce the dose to avoid hypoglycemias, ${ }^{49}$ especially when there is weight loss, nausea, and vomiting. ${ }^{2}$ Premixes of insulin, intermediate-acting insulin or long-acting analogs should be adjusted to the needs ${ }^{45}$ preferring the use of insulin analogs since they show a lower risk of hypoglycemia. ${ }^{52}$ The basal scheme boluses and infusion pumps are very useful especially when there is nausea or vomiting; since it is possible to adjust the dose according to the amount and times of the patient's food. ${ }^{29}$ New drugs used in type 1 diabetes, such as incretin analogs, inhibitors of $\mathrm{SGTL}_{2}$ or inhibitors of $\mathrm{DDP}_{4}{ }^{52}$ must be adjusted according to the characteristics of the patients or even discontinued. ${ }^{48}$

\section{Patients with type 2 diabetes}

Type 2 diabetes is characterized by insulin resistance and impaired insulin secretion and, although its initial treatment includes diet and lifestyle changes plus oral antidiabetics, in some patients it is necessary to use insulin or other injectables to reach glycemic control. ${ }^{53}$ Patients with type 2 diabetes have a lower risk of complications such as hypoglycemia and ketoacidosis compared to patients with type 1 diabetes. ${ }^{51}$ The main difficulty of hyperglycemia is the hyperosmolar state $^{54}$ with higher risk in older adults, ${ }^{14}$ so the adjustment of medications in end-of-life care is essential. ${ }^{50}$ Treatment with insulin could be simplified or discontinued if symptomatic hypoglycemia occurs and according to clinical status, ${ }^{45}$ with attention in patients with clinical and unstable detergency and those close to death. ${ }^{46}$ Many oral drugs are associated with hypoglycemia. ${ }^{23}$ Others with nausea, reduced appetite or must be modified depending on the glomerular function. ${ }^{55}$ Drugs such as linagliptin and repaglinide may be useful, ${ }^{2}$ however the need to be laxer in the treatment schemes; simplifying schemes is favorable to increase the quality of life and reduce the risk of side effects of drugs. ${ }^{45}$ The same occurs for other medications that are usually used for the comorbidities that accompany diabetes. ${ }^{48}$

Many patients with diabetes develop some cancer-related to multiple mechanisms ${ }^{56}$ and this increases mortality in patients with terminal cancer by different mechanisms when compared with nondiabetic patients. ${ }^{4}$ Many cancer patients are managed therapeutically with steroids, which is related to greater difficulty in reaching glycemic goals, ${ }^{2}$ which makes complex the management of these patients. ${ }^{4}$ Therefore, when using steroids, we must consider the riskbenefit considering the use of low doses to avoid complications ${ }^{2}$ and adjust the treatment according to the characteristics of the patient and their glucose monitoring. ${ }^{48}$

\section{Withdrawal of treatment}

There is no evidence of the actions to be taken regarding suspending treatment in terminally ill patients. ${ }^{29}$ Multiple factors influence this process ${ }^{48}$ Many ethical issues are involved at the time of deciding to suspend or not initiate any treatment. ${ }^{29}$ Determining the forecast is a significant step in this situation and helps to determine the changes. ${ }^{49}$ However, it is a complicated process. ${ }^{29}$ The American 
Diabetes Association describes three scenarios through which we can make general decisions about the changes in the management and treatment of patients, referring that in the case of patients who are close to death is due to consider the withdrawal of some therapies. ${ }^{34}$ Some factors suggest a bad prognosis in certain individual ${ }^{29,45}$ (Table 3) The GSF can be useful for making decisions in these cases. ${ }^{36}$

Table 3 Indicators of poor prognosis

Presence of comorbidities

Unintentional loss of weight of $>10 \%$ in a short period (six months)

Functional deterioration

Serum albumin $<2.5 \mathrm{~g} / \mathrm{L}$

Need for help for daily living activities and self-care of diabetes

We can consider the following conditions to make treatment withdrawal decisions: ${ }^{48}$

i. When individuals are entering the final stage of life

ii. When frequent hypoglycemia related to medications is making it difficult to manage

iii. When it is not possible to justify the benefits of strict glucose control

iv. When the control treatment of blood pressure or other drugs are not justified by the risk that condition

v. When the person decides not to choose to continue with the treatment of food or fluids

vi. When continuing with insulin presupposes a risk higher than the benefit

\section{Conclusion}

No one is prepared to die. Therefore, it is necessary to consider the inclusion of palliative care early in diabetes. Strengthen multidisciplinary care strategies to avoid fragmentation and work integrating the patient and the family to make informed decisions favoring person-centered care. It is important to use existing tools to identify early on those patients who will benefit from palliative care as well as identify those who enter the end-of-life care settings and terminals with the objective of providing effective treatment to avoid stubbornness therapeutically and help keep these patients comfortable and with the improved quality of life.

\section{Acknowledgements}

I want to thank Antonio Rodríguez González. Silex-Solutions for your support.

\section{Conflict of interests}

The author declares no conflict of interest.

\section{References}

1. IDF. IDF diabetes Atlas. 8th ed. IDF; 2017. 150 p.

2. Claydon A, Spencer K. Improving diabetes care at the end of life. Nurs Stand. 2015;30(6):37-42.

3. Dunning T, Martin P. Palliative and end of life care of people with diabetes: Issues, challenges and strategies. Diabetes Res Clin Pract. 2017;S0168-8227(17)31269-X.
4. Kondo S, Kondo M, Kondo A. Glycemia control using A1C level in terminal cancer patients with preexisting type 2 diabetes. J Palliat Med. 2013;16(7):790-793.

5. Dunning T, Duggan N, Savage S. Caring for people with diabetes at the end of life. Curr Diab Rep. 2016;16(11):103.

6. Johnston GM, Lethbridge L, Talbot P, et al. Identifying persons with diabetes who could benefit from a palliative approach to care. Can $J$ Diabetes. 2015;39(1):29-35.

7. Rao Kondapally Seshasai S, Kaptoge S, Thompson A, et al. Diabetes mellitus, fasting glucose, and risk of cause-specific death. $N$ Engl J Med. 2011;364(9):829-841.

8. Dunning T. Integrating palliative care with usual care of diabetic foot wounds. Diabetes Metab Res Rev. 2016;32 Suppl 1:303-310.

9. Sarwar N, Gao P, Seshasai SR, et al. Diabetes mellitus, fasting blood glucose concentration, and risk of vascular disease: A collaborative metaanalysis of 102 prospective studies. Lancet. 2010;375(9733):2215-2222.

10. Tancredi M, Rosengren A, Svensson A-M, et al. Excess mortality among persons with type 2 diabetes. $N$ Engl J Med. 2015;373(18):1720-1732.

11. Baena-Díez JM, Peñafiel J, Subirana I, et al. Risk of cause-specific death in individuals with diabetes: a competing risks analysis. Diabetes Care. 2016;39(11):1987-1995.

12. Dionisio R, Giardini A, Cata P de, et al. Diabetes management in end of life: a preliminary report stemming from clinical experience. Am J Hosp Palliat Care. 2015;32(6):588-593.

13. Kirkman MS, Briscoe VJ, Clark N, et al. Diabetes in older adults: A consensus report. J Am Geriatr Soc. 2012;60(12):2342-2356.

14. Munshi MN, Florez H, Huang ES, et al. Management of diabetes in long-term care and skilled nursing facilities: a position statement of the american diabetes association. Diabetes Care. 2016;39(2):308-318.

15. Hunt KJ, May CR. Managing expectations: Cognitive authority and experienced control in complex healthcare processes. BMC Health Serv Res. 2017;17(1):459.

16. Domínguez-Marín AD. Cuidados paliativos. Sus orígenes y su llegada a México. Rev Hosp Jua Mex. 2016;83(3):64-65.

17. Panagiotopoulos C, Hadjiyannakis S, Henderson M. Type 2 diabetes in children and adolescents. Can J Diabetes. 2018;42(Suppl 1):S247-254.

18. Lynn J, Adamson DM. Living well at the end of life: Adapting health care to serious chronic illness in old age. White paper, WP-137. Santa Monica, California: Rand; 2003. p. III-19.

19. Giovannucci E, Harlan DM, Archer MC, et al. Diabetes and cancer: a consensus report. Diabetes Care. 2010;33(7):1674-1685.

20. Johnson JA, Carstensen B, Witte D, et al. Diabetes and cancer (1): Evaluating the temporal relationship between type 2 diabetes and cancer incidence. Diabetologia. 2012;55(6):1607-1618.

21. Johnson JA, Pollak M. Insulin, glucose and the increased risk of cancer in patients with type 2 diabetes. Diabetologia. 2010;53(10):2086-2088.

22. Goguen J, Gilbert J. Hyperglycemic emergencies in adults. Can $J$ Diabetes. 2018;42 Suppl 1:S109-114.

23. Morales J, Schneider D. Hypoglycemia. Am J Med. 2014;127(10 Suppl):S17-24.

24. CENETEC. Diagnóstico y tratamiento del síndrome de fragilidad en el adulto mayor. Guía de referencia rápida: CENETEC; 2014. p. 6-49.

25. Strain WD, Hope SV, Green A, et al. Type 2 diabetes mellitus in older people: A brief statement of key principles of modern day management 
including the assessment of frailty. A national collaborative stakeholder initiative. Diabet Med. 2018;35(7):838-845.

26. Sinclair A, Dunning T, Colagiuri S. Managing older people with type 2 diabetes. Global guideline. Bruselas, Bélgica: International diabetes federation; 2013. $94 \mathrm{p}$

27. Hanna JS. Sarcopenia and critical illness: A deadly combination in the elderly. JPEN J Parenter Enteral Nutr. 2015;39(3):273-281.

28. World Health Organisation. Palliative care is an essential part of cancer control. Switzerland: WHO; 2014.

29. Dunning T, Savage S, Duggan N, et al. Palliative and end of life care for people with diabetes: A topical issue. Diabetes Management. 2014;4(5):449-460.

30. Tapley M, Needham E, Hoey L. 'End of Life Diabetes Care: A Strategy Document': where are we one year on in the light of the review of the Liverpool Care Pathway? Practical Diabetes. 2013;30(9):355-356.

31. Claessen SJJ, Francke AL, Engels Y, et al. How do GPs identify a need for palliative care in their patients? An interview study. BMC Fam Pract. 2013;14:42.

32. Wilkinson E, Randhawa G, Brown E, et al. et al. Exploring access to end of life care for ethnic minorities with end stage kidney disease through recruitment in action research. BMC Palliat Care. 2016;15:57.

33. Emanuel L, Alexander C, Arnold RM, et al. Integrating palliative care into disease management guidelines. J Palliat Med. 2004;7(6):774-783.

34. American Diabetes Association. 11. Older adults. standards of medica care in diabetes-2018. Diabetes Care. 2018;41(Suppl 1):S119-125.

35. Thomas K. Gold Standards Framework Centre. Prognostic indicator guidance. 4th ed. UK: Gold Standards Framework Centre; 2011

36. Thomas J, GSF Team. The Gold Standards Framework Proactive Identification Guidance (PIG): The National GSF Centre's guidance for clinicians to support earlier identification of patients nearing the end of life leading to improved proactive person-centred care. 6th ed. UK: The Gold Standards Framework; 2016

37. Barnett K, Mercer SW, Norbury M, et al. Epidemiology of multimorbidity and implications for health care, research, and medical education: A cross-sectional study. Lancet. 2012;380(9836):37-43.

38. Smith SM, Soubhi H, Fortin M, et al. Managing patients with multimorbidity: Systematic review of interventions in primary care and community settings. BMJ. 2012;345:e5205

39. Bernabeu-Wittel $\mathrm{M}$, Alonso-Coello $\mathrm{P}$, Rico-Blázquez $\mathrm{M}$, et al Desarrollo de guías de práctica clínica en pacientes con comorbilidad y pluripatología. Aten Primaria. 2014;46(7):385-392.

40. Li Xueqing, Kou Shunchao, Blanco Aspiazu Miguel Ángel. Abordaje de los mecanismos de comorbilidad en diabetes mellitus tipo 2. Rev haban cienc méd. 2017;16(6):891-901.
41. Bernabeu-Wittel M, Ollero-Baturone M, Moreno-Gaviño L, et al. Development of a new predictive model for polypathological patients. The PROFUND index. Eur J Intern Med. 2011;22(3):311-317.

42. Colombo PB, Nieto Martín MD, La Pascual de Pisa B, et al. Validación de un modelo pronóstico para pacientes pluripatológicos en atención primaria: Estudio PROFUND en atenci n primaria. Atención Primaria. 2014;46(3):41-48.

43. Budge P. Management of diabetes in patients at the end of life. Nursing Standard. 2010;25(6):42-46.

44. Pfaff K, Markaki A. Compassionate collaborative care: An integrative review of quality indicators in end-of-life care. BMC Palliat Care. 2017;16(1):65.

45. Rowles S, Kilvert A, Sinclair A. ABCD position statement on diabetes and end of life care. Pract Diab Int. 2011;28(1):26-27.

46. Diabetes UK. End of Life Diabetes Care: Full Strategy.

47. American Diabetes Association. 6. Glycemic Targets: Standards of Medical Care in Diabetes-2018. Diabetes Care. 2018;41(Suppl 1):S5564

48. Diabetes UK. End of life diabetes care: Clinical Care Recommendations. 2013.

49. Angelo M, Ruchalski C, Sproge BJ. An approach to diabetes mellitus in hospice and palliative medicine. J Palliat Med. 2011;14(1):83-87.

50. Lindskog M, Kärvestedt L, Fürst CJ. Glycaemic control in end-of-life care. Curr Opin Support Palliat Care. 2014;8(4):378-382.

51. Tourkmani AM, Alharbi TJ, Rsheed AMB, et al. Hypoglycemia in Type 2 Diabetes Mellitus patients: A review article. Diabetes Metab Syndr. 2018;S1871-4021.

52. Iqbal A, Novodvorsky P, Heller SR. Recent Updates on Type 1 Diabetes Mellitus Management for Clinicians. Diabetes Metab J. 2018;42(1):318

53. Vargas-Uricoechea H. Efficacy and Safety of Insulin Glargine $300 \mathrm{U} / \mathrm{mL}$ versus $100 \mathrm{U} / \mathrm{mL}$ in Diabetes Mellitus: A Comprehensive Review of the Literature. Journal of Diabetes Research. 2018;2018:2052101.

54. Adeyinka A, Kondamudi NP. Hyperosmolar Hyperglycemic Nonketotic Coma (HHNC, Hyperosmolar Hyperglycemic Nonketotic Syndrome). Treasure Island: StatPearls; 2018.

55. Thrasher J. Pharmacologic Management of Type 2 Diabetes Mellitus: Available Therapies. Am J Med. 2017;130(6S):S4-17.

56. Ballotari P, Vicentini M, Manicardi V, et al. Diabetes and risk of cancer incidence: Results from a population-based cohort study in northern Italy. BMC Cancer. 2017;17(1):703. 